\title{
Doping mechanisms and impedance study of Ba-substituted bismuth magnesium niobate pyrochlores
}

\begin{abstract}
Here we investigated the doping mechanisms and electrical properties of Ba-substituted Bi3.36Mg1.92-xBaxNb2.72O14.36 (BMBN) pyrochlores. Phase-pure BMBN pyrochlores prepared by solid-state reaction were found to crystallise in a cubic structure (Fd3m; No. 227) with their lattice parameters in the range 10.5968(16) 10.5879(3) A. The Scanning Electron Microscopy (SEM) analysis revealed that BMBN pyrochlores were composed of irregular shaped grains and their estimated crystallite sizes by both Williamson-Hall and Scherrer methods were in the range 50-75 nm and 60-70 nm, respectively. Neither thermal event nor significant weight loss was observed over the studied temperature range $\sim 30-1000{ }^{\circ} \mathrm{C}$, confirming that BMBN pyrochlores were thermally stable with negligible $\mathrm{Bi} 2 \mathrm{O} 3$ loss. The high activation energies in the range $1.18-1.30 \mathrm{eV}$, as determined by the Arrhenius conductivity plots, showed BMBN pyrochlores to be highly insulating. The dielectric constants, $\varepsilon^{\prime}$ and dielectric losses, $\tan \delta$ were found in the range $147-183$ and $7.9 \times 10-2-9.6 \times 10-2$ at $\sim 30{ }^{\circ} \mathrm{C}$ and 1 MHz. Negative temperature coefficient of capacitances, TCC, in the range $(-478)-$ (-688) $\mathrm{ppm} /{ }^{\circ} \mathrm{C}$ were recorded over $\sim 30-300{ }^{\circ} \mathrm{C}$ at $1 \mathrm{MHz}$.
\end{abstract}

Keyword: Pyrochlore; Solid-state reaction; Doping mechanism; Dielectric; Impedance spectroscopy 